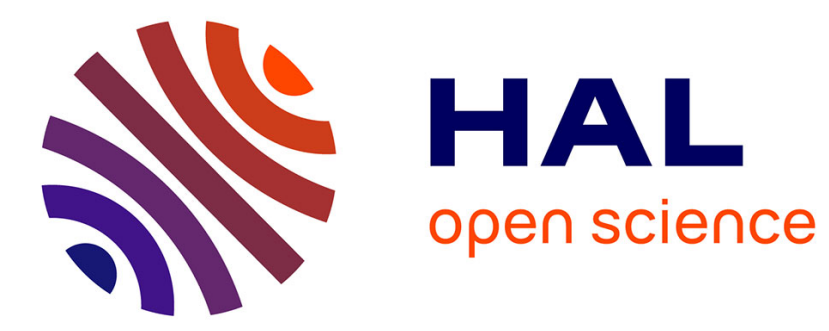

\title{
Establishing Accuracy of Watershed-Derived Pore Network Extraction for Characterizing In-Plane Effective Diffusivity in Thin Porous Layers
}

\author{
Stéphane Chevalier, J. Hinebaugh, A. Bazylak
}

\section{To cite this version:}

Stéphane Chevalier, J. Hinebaugh, A. Bazylak. Establishing Accuracy of Watershed-Derived Pore Network Extraction for Characterizing In-Plane Effective Diffusivity in Thin Porous Layers. Journal of The Electrochemical Society, 2019, 166 (7), pp.F3246-F3254. 10.1149/2.0251907jes . hal-02924016

\author{
HAL Id: hal-02924016 \\ https://hal.science/hal-02924016
}

Submitted on 31 Aug 2020

HAL is a multi-disciplinary open access archive for the deposit and dissemination of scientific research documents, whether they are published or not. The documents may come from teaching and research institutions in France or abroad, or from public or private research centers.
L'archive ouverte pluridisciplinaire HAL, est destinée au dépôt et à la diffusion de documents scientifiques de niveau recherche, publiés ou non, émanant des établissements d'enseignement et de recherche français ou étrangers, des laboratoires publics ou privés. 


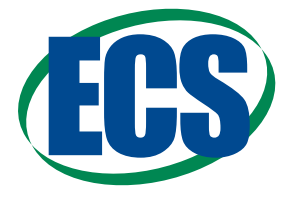

Jes focus Issue on Advances in Modern Polymer Electrolyte Fuel Cells in Honor of Shimshon Gottesfeld

\title{
Establishing Accuracy of Watershed-Derived Pore Network Extraction for Characterizing In-Plane Effective Diffusivity in Thin Porous Layers
}

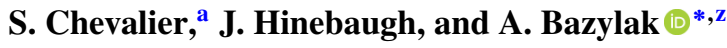 \\ Thermofluids for Energy and Advanced Materials (TEAM) Laboratory, Department of Mechanical \& Industrial \\ Engineering, University of Toronto Institute for Sustainable Energy, Faculty of Applied Science \& Engineering, \\ University of Toronto, Toronto, Ontario M5S 3G8, Canada
}

The work presented in this paper reports a versatile and customizable watershed-based pore network extraction tool. It was used to build pore networks from microscale computed tomography images of highly porous carbon-fiber materials. The extracted networks were used to characterize the structural properties (pore and throat diameter, and porosity distributions) and to predict the dry, in-plane, effective diffusivities of two commercial gas diffusion layers under various levels of compression. The relationship between compression and effective diffusivity was used to accurately predict experimental values available in the literature to within $10 \%$ of the reported values for compressive strains less than 0.55 . Through this work, we validate the accuracy of watershed-derived pore networks for predicting in-plane effective diffusivities, further establishing the reliability of watershed-derived pore network modeling for fuel cell applications.

(C) The Author(s) 2019. Published by ECS. This is an open access article distributed under the terms of the Creative Commons Attribution Non-Commercial No Derivatives 4.0 License (CC BY-NC-ND, http://creativecommons.org/licenses/by-nc-nd/4.0/), which permits non-commercial reuse, distribution, and reproduction in any medium, provided the original work is not changed in any way and is properly cited. For permission for commercial reuse, please email: oa@electrochem.org. [DOI: 10.1149/2.0251907jes]

(cc) BY-NC-ND

Manuscript submitted January 7, 2019; revised manuscript received March 12, 2019. Published May 6, 2019. This paper is part of the JES Focus Issue on Advances in Modern Polymer Electrolyte Fuel Cells in Honor of Shimshon Gottesfeld.

Polymer electrolyte membrane (PEM) fuel cells enable the use of hydrogen as a renewable energy carrier to a broad range of applications, including transportation and mobile power stations. By increasing the power density of the PEM fuel cell, smaller and more cost-effective systems can be manufactured. In order to boost peak power density, the efficiency levels at high current density operation must increase. The dominating losses at high current density operation are mass transport losses, which can be described as quantifying the insufficient transport of reactants to the active reaction sites. ${ }^{1-3}$ This issue is currently one of the key limiting factors hindering the development of high power density fuel cells. ${ }^{4-6}$

A porous, conductive material, referred to as the gas diffusion layer (GDL) or otherwise known as the porous transport layer (PTL) or diffusion media (DM), lies between the channels and the catalyst layers at anode and cathode. The GDL is responsible for evenly distributing the supplied reactants across the catalyst layer, transporting the byproducts (water) from the catalyst layer to the flow field, and providing electrical pathways between the catalyst layer and the flow field. The diffusivity of the GDL governs the $\mathrm{O}_{2}$ arrival rates at the catalyst layer. Therefore, the careful manipulation of the diffusive properties of the GDL is a current research focus for the industry. As evidenced by the number of years that this issue has remained a top research focus, ${ }^{7-13}$ the optimization of GDL properties has turned out to be a difficult problem to study because the diffusive properties of the GDL change during operation (i.e., the appearance and accumulation of liquid water within the GDL ${ }^{14,15}$ ). What may seem like a highly porous GDL architecture, ideal for gas diffusion, may also be one that easily floods with liquid water.

Due to the complexity of the system, PEM fuel cell manufacturers may be compelled to adopt a trial and error approach when choosing GDL materials. One response to this has been the development of pore network models applied to GDLs. This approach allows the prediction of both liquid water configurations as well as the

\footnotetext{
*Electrochemical Society Member.

${ }^{\text {a }}$ Present Address: Institut de Mécanique et d'Ingénierie de Bordeaux (CNRS UMR

5295), ENSAM, Esplanade des Arts \& Métiers 33405 Talence, France.

${ }^{\mathrm{z}}$ E-mail: abazylak@mie.utoronto.ca
}

diffusive properties of the GDL in such wet states of operation. ${ }^{16-20}$ Unlike traditional continuum models, pore network models involve a domain that is made up of a network of discrete pores, which may become partially or fully saturated with liquid water. The local diffusivity characteristics of the material significantly depend on the degree to which these pores are saturated with liquid water. While many pore network modelling studies have provided important information pertaining to the relationship between 3D morphological structures and material diffusive properties for GDL-like porous materials, ${ }^{21-24}$ only few studies have additionally provided comparisons of GDL morphologies..$^{25,26}$ The determination of effective transport properties for fuel cell GDL materials is contingent upon the creation of materialspecific pore networks instead of general networks with only GDL-like properties.

The combination of the powerful simulation capabilities of pore network modelling along with the computed tomography (CT) provides an ideal method for studying GDL morphologies. This approach was used by Agaesse et al. ${ }^{27}$ to model two-phase flow transport in fuel cell GDLs, and their modelling results were in excellent agreement with their experimental data. Effective diffusivity calculations based on microscale CT images were performed by Zenyuk et al., ${ }^{28}$ who used an alternative method to pore network modeling - finite-element computation. In his work, Gostick ${ }^{29}$ reported a watershed segmentation algorithm for generating a pore network from CT images. Although his work showed that extracted networks can be used to analyze the structural properties of several porous materials, the absolute values of the permeability tensor were not characterized. Therefore, improvements to watershed-based network extractions as well as additional experimental validation (based on transport material properties, such as effective diffusivity) are still needed.

Effective diffusivity characterizations of fuel cell GDLs have been extensively reported over the last two decades ${ }^{30,31}$ using various in situ experimental techniques, such as limiting current, ${ }^{32}$ electrochemical impedance spectroscopy. ${ }^{10,33}$ Fuel cell GDL effective diffusivity has been also characterized ex situ using a large variety of methods, such as electrochemical diffusimetry, ${ }^{34}$ or based onto diffusion cells. ${ }^{35-37}$ Numerical simulations of mass transport through the GDL are also valuable alternatives to characterizing the GDL effective diffusivity, such as the work reported by García-Salaberri et al. ${ }^{38}$ using Lattice 


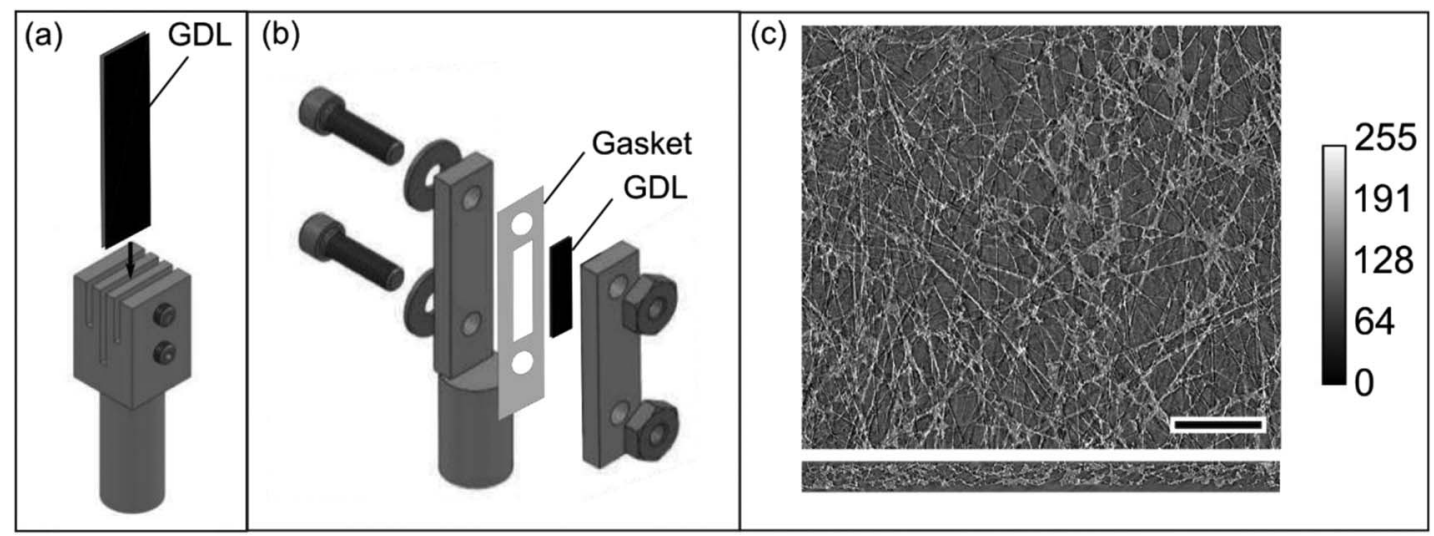

Figure 1. (a), Uncompressed material holder. (b), Compressed material holder. (c), Through-plane (top) and IP view (bottom) of a raw CT scan of the SGL $34 \mathrm{AA}$ GDL. The length of the scale bar is $1 \mathrm{~mm}$.

Boltzmann method. However, new GDL materials are continuously reported in the literature, ${ }^{39}$ and therefore these materials need to be accurately characterized to assess their performance. However, all of the above-mentioned studies have been focused on the through-plane direction, as diffusion in this direction dominates the rate of reactant supply to the electrode. Fibrous materials like GDLs possess significant structural anisotropy due to the orientation of the fibers, so to fully characterize these materials, it is necessary to measure their effective diffusivity tensor. Diffusion in the in-plane (IP) direction is a critical factor: this information is needed for 3D modeling studies of gas distribution and catalyst utilization within the cell, and the IP diffusivity also impacts engineering decisions such as the optimal channel-to-rib ratio and GDL thicknesses.

Surprisingly, few studies have been performed on GDLs in the IP direction. To date, the only IP diffusivity measurements reported in literature were performed by Rashapov et al. ${ }^{36,40}$ and Kramer et al., ${ }^{41}$ which involved the development of a specific experimental apparatus. Characterizing the effective diffusivity of the GDL based on computed tomography or numerical generations via pore network modeling provides a tremendous opportunity for fuel cell designers to rapidly propose and evaluate candidate materials. Pore network modelling becomes particularly attractive when watershed-derived pore networks are used since they inherently capture the deterministic, irregular, true spatial distributions of material solid and void spaces. Indeed, pore network modelling for fuel cell applications has attracted significant attention in recent years; however, there is a notable scarcity in validation work for pore network modelling that is needed to establish the capabilities of this approach for the fuel cell sector. In particular, the accuracy of in-plane effective diffusivity is one key property that must be firmly established due to the importance of lateral rib-channel transport mechanisms in the fuel cell.

In this study, we propose a fully customizable methodology to extract pore networks from CT images. This method is based on watershed-derived pore network modelling, and it can be used to compute the IP effective diffusivities using the open source pack- age OpenPNM. ${ }^{16}$ The code is validated using the experimental IP effective diffusivities reported by Rashapov et al. ${ }^{36}$ for two sets of GDLs (SGL 25AA and 34AA) over a range of compression ratios (or compressive strain). The focus of this study is to characterize the IP effective diffusivity, since little attention has been paid to this GDL property despite its influence on how to select an optimal channel-to-rib ratio and GDL thickness. Herein, only one IP direction was chosen since both IP directions have the same transport properties. $^{29}$

\section{Methodology}

CT and segmentation.-The GDL samples considered in this work (SGL 25AA and SGL 34AA) were examined across a range of compression ratios. Each GDL sample was made of a fibrous carbon paper without an MPL. The detailed, 3D structures were obtained using an X-ray-based microscale CT scanner (Skyscan 1172). The pixel resolution of the radiographs ranged between $2.88 \mu \mathrm{m} / \mathrm{px}$ and $3.23 \mu \mathrm{m} / \mathrm{px}$. The sample areas were $5 \times 5 \mathrm{~mm}^{2}$ for the uncompressed samples and $4.0 \times 1.5 \mathrm{~mm}^{2}$ for the compressed samples. To obtain uncompressed and compressed GDL structures, the GDLs were held with a custom setup as shown in Figures 1a and 1b, respectively. To image the uncompressed GDL structure, the GDL sheet was held on a single edge, while the uncompressed region was imaged. The compressed GDL was imaged using the holder presented in Figure 1b, and the compression ratio was controlled using stacked polyethylene naphthalate (PEN) sheets. The SGL 25AA and SGL 34AA GDLs were imaged for a range of compression ratios. For each compression ratio used, the associated strain was computed as the difference between the uncompressed thickness, $l_{o}$, and the compressed thickness, $l$, divided by the uncompressed thickness as follows: strain $=\left(l_{0}-l\right) / l_{o}$. Table I summarizes all the imaged samples with their associated compression ratios, strains, and compressed thicknesses.

Examples of the IP and through-plane greyscale images of the 3D reconstructed GDL (obtained through CT imaging) are presented in

Table I. Characteristics of the GDL samples used and the associated thicknesses.

\begin{tabular}{|c|c|c|c|c|c|c|c|c|c|}
\hline Name & \multicolumn{4}{|c|}{ SGL 25AA } & & \multicolumn{4}{|c|}{ SGL 34AA } \\
\hline Bulk porosity & \multicolumn{4}{|c|}{0.92} & & \multicolumn{4}{|c|}{0.88} \\
\hline Areal weight $\left(\mathrm{g} / \mathrm{m}^{2}\right)$ & \multicolumn{4}{|c|}{38} & & \multicolumn{4}{|c|}{77} \\
\hline Uncompressed thickness $(\mu \mathrm{m})$ & \multicolumn{4}{|c|}{$178^{36}$} & & \multicolumn{4}{|c|}{$252^{36}$} \\
\hline Thicknesses studied $(\mu \mathrm{m})$ & 178 & 100 & 75 & 50 & 252 & 200 & 150 & 125 & 100 \\
\hline Strain & 0.00 & 0.44 & 0.58 & 0.72 & 0.00 & 0.21 & 0.40 & 0.50 & 0.60 \\
\hline
\end{tabular}


Figure 1c. We used an in-house segmentation algorithm similar to Ref. 42 to carefully distinguish between the solid and void phases. The algorithm was used to threshold the greyscale images based on the fiber density computed as:

$$
\rho_{f}=\frac{\sigma_{w}}{l_{0}\left(1-\varepsilon_{0}\right)}
$$

where $\sigma_{w}$ is the areal weight of the sample, and $\varepsilon_{0}$ is the uncompressed porosity of the sample given by the manufacturer (SGL Sigracet, see Table I). Small variabilities in GDL porosity values have been reported in past literature ${ }^{43}$ however, these variabilities do not exceed $\pm 5 \%$ (for example Rashapov et al. ${ }^{44}$ measured 0.884 and 0.841 for SGL 25AA and 34AA, respectively). We assume that these small variations in porosity would only impact a few voxels and not affect the global segmentation result.

In order to compute the fiber density, we measured areal weight of the GDL. The areal weight was measured to be $38 \mathrm{~g} / \mathrm{m}^{2}$ and $77 \mathrm{~g} / \mathrm{m}^{2}$ for the SGL 25AA and SGL 34AA GDLs, respectively. They were measured by determining the masses of GDL samples with known dimensions. One to six GDL samples were cut using a 1 inch-punch and stacked together to measure their cumulative masses.

Finally, once the images were segmented, a 3D median filter with a radius of 1 voxel was used to clean the binary images.

The GDL porosity was computed based on the binary image obtained after segmentation. The number of solid voxels $(S V)$ - consisting of carbon paper fibers - and void voxels $(V V)$ were summed at each through-plane position, and the porosity distribution was computed as follows:

$$
\varepsilon(z)=\frac{V V}{S V+V V}
$$

where $\varepsilon$ is the GDL porosity, and $z$ is the through-plane direction.
Extraction algorithm.-Similar to the Maximal Ball Method $(\mathrm{MBM})$ presented by Dong and Blunt, ${ }^{45}$ the algorithm employed in this work was initiated with a distance map of a binary pore space, populated with the Euclidean distances between each pore voxel and its nearest solid voxel. Similar to the MBM, the local maxima in this distance map were assumed to be pore centers, as these are the locations and radii of the largest possible spheres that could occupy the space. However, unlike the MBM, the pore space was divided using a customized Watershed ${ }^{46}$ algorithm on the inverse of the distance map. A set of 3D matrices of identical shapes were generated as described below. These matrices, once generated, were accessed at various points in the network extraction algorithm (Procedure 1, below). While the algorithms discussed were applied to 3D datasets, the figures accompanying the following sections were created in 2D for illustrative clarity.

The extraction algorithm presented here is admittedly slower than similar algorithms, which take advantage of highly optimized watershed sub-algorithms (a great example of which was presented by Gostick $^{29}$ ). We utilized the following algorithm written from the ground up in efficient languages, such as $\mathrm{C}++$ combined with Matlab for the interface, thereby providing a highly customizable platform to add rules for preventing pore over- or under-segmentation at any point in the process. We believe this flexibility may be of use as the field of CT derived pore networks expands.

Binary matrix.-The box-shaped binary domain was first oriented such that one pair of opposing faces, labelled "top" and "bottom", of the six sided domain corresponded to the inlet and outlet faces for any intended pore network simulation. The other four walls were then padded with solid voxels in order to simulate a solid enclosure around the sample. This binary matrix was labelled $\mathbb{B}$, where $\mathbb{B}(i)=1$, if (a)
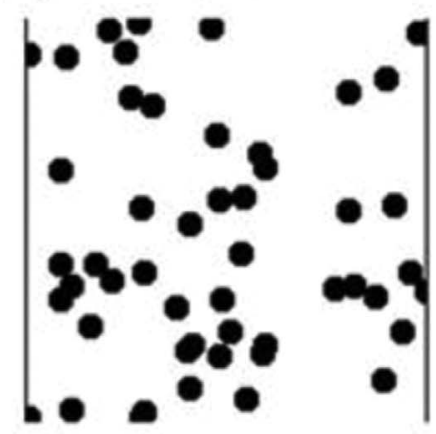

(d)

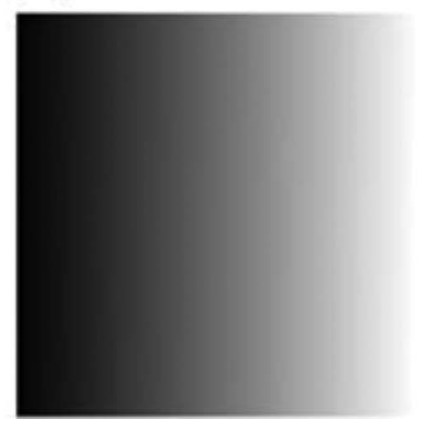

(b)
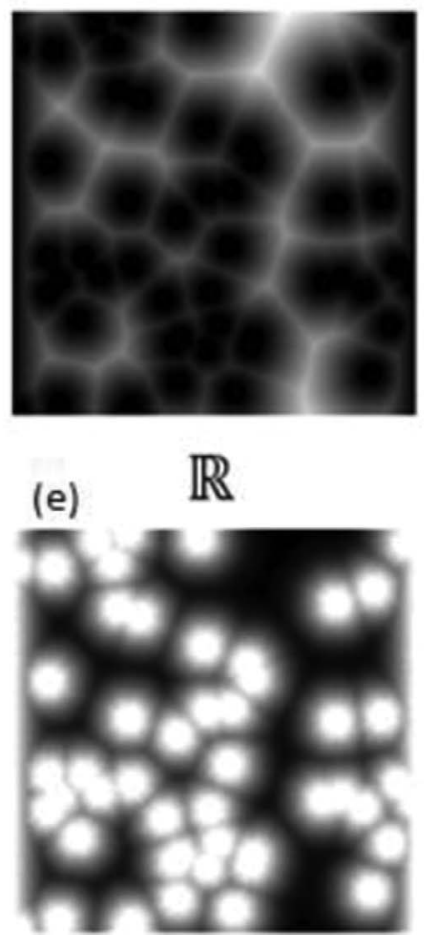

(c)
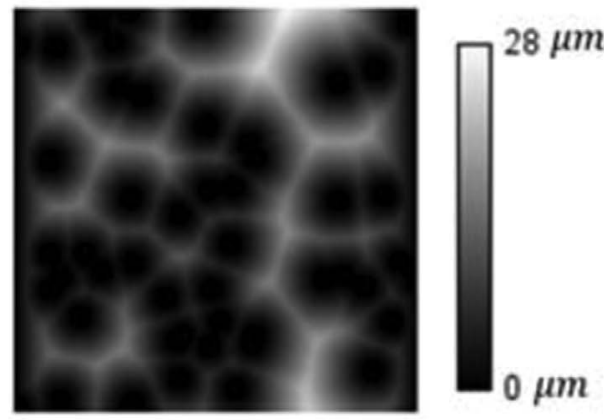

(f)

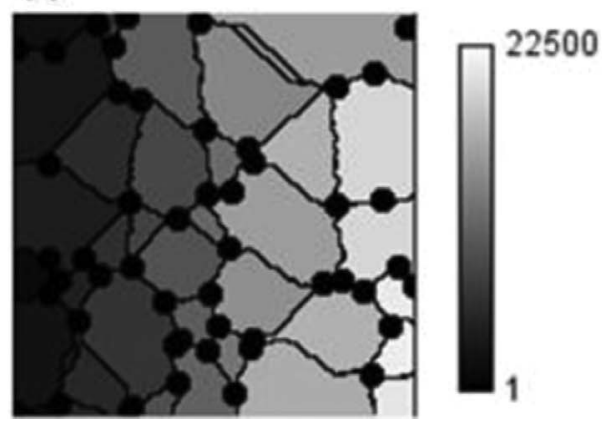

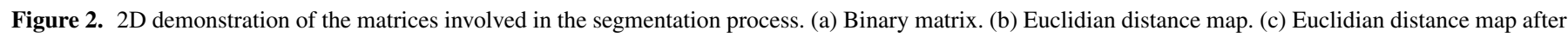
the median filter. (d) Index matrix. (e) Ranking matrix. (f) Pore space matrix. 
voxel $i$ is solid (shown as black in Figure 2), and $\mathbb{B}(i)=0$, if voxel $i$ is void (shown as white in Figure 2).

In some circumstances, such as with stochastically modelled materials, it may be useful to generate an extracted pore network with periodic side-wall boundary conditions. In these cases, instead of a padding of solid voxels, the four side-walls may be padded with a number of voxel planes from the opposite face of the domain. The pores may then be stitched together at the end of the extraction process.

Index matrix.-A new matrix was next formed with the same shape as the binary matrix; however, instead of 0 s and 1 s, the matrix was filled with the linear index value for each voxel position, where the $n$ voxels were each assigned an index between 0 and $n-1$, creating matrix $\mathbb{I}$. It is useful to index $\mathbb{I}$, such that $\mathbb{I}(i)=i$. In this process sometimes referred to as "flattening", we can now treat an $L \mathrm{x} W \mathrm{x} H$ matrix as an $\left(L^{*} W^{*} H\right) \mathrm{x} 1$ array.

Distance matrices.-A Euclidean distance map, $\mathbb{D}$, was defined using the bwdist function from the MATLAB Image Processing Toolbox. ${ }^{47}$ In this matrix, $\mathbb{D}(i)=0$, if voxel $i$ was a solid, and $\mathbb{D}(i)=d$, if voxel $i$ was a void, where $d$ was the Euclidean distance between the center of voxel $i$ and the center of the nearest solid voxel.

While the pore and throat radii were ultimately found from $\mathbb{D}$, a 3D median filter (using a spherical kernel with radius of 2 voxels) was applied to $\mathbb{D}$, resulting in $\mathbb{D}$. This filtering process temporarily suppressed the sharp features within $\mathbb{D}$, which prevented an unnecessary oversegmentation of the pore space.

Matrix for ranking voxels. - A new matrix, $\mathbb{R}$, which ranked the voxels according to their $\mathbb{D}$ value in descending order was generated (lowest number has the highest rank). In the case where multiple voxels had identical $\mathbb{D}$ values, the rank was decided based on a random permutation of the set of like voxels. This process provided each voxel with a unique, integer value for $\mathbb{R}$ between 0 and $n-1$, and the random permutation prevented a directional bias during extraction.

Segmented pore matrix.-The matrix that eventually stores the segmented pore space, labelled $\mathbb{P}$, was initiated with a duplicate of $\mathbb{I}$ and modified such that all solid voxels $(\mathbb{B}=1)$ were replaced with a value of -1 . After the pore segmentation process, the void voxels within $\mathbb{P}$ were populated with pore names.

Pore segmentation algorithm.--In this algorithm, each pore name was defined by the index, $i$, of the ancestor voxel of that pore. The ancestor voxel is the highest-ranking voxel in that pore, meaning that it has the property that $\mathbb{R}(i)<\mathbb{R}(j)$ for all voxels, $j$, in the pore. Due to the definition of $\mathbb{R}$, this resulted in ancestors with maximal distance values (i.e. $\mathbb{D}(i) \geq \mathbb{D}(j)$ for all voxels $j$ in the pore).

The global algorithm followed the logic described hereafter. Initially, all void voxels were defined as their own ancestors (i.e., $\mathbb{P}=\mathbb{I}$ ). Then, as the focus voxel, $i$, was incremented from the $0^{\text {th }}$ rank (i.e. $\mathbb{R}(i)=0)$ to the $n-1^{\text {th }}$ rank, void neighbors were evaluated. Each neighboring void voxel, $j$, could potentially change its ancestor value to that of the focus (i.e., $\mathbb{P}(j)$ can become $\mathbb{P}(i)$ ), if $\mathbb{R}(i)<\mathbb{R}(j)$, (i.e., $\mathbb{D}(i) \geq \mathbb{D}(j))$.

In some cases, (along a longitudinally symmetric tube, for example) a string of equal $\mathbb{D}$ values could exist whereby a number of segmented pores could be created, depending on the randomized rank of each of the central voxels. In such an instance, these additional pores would be collapsed into a single pore. This was achieved by checking whether the ancestor's distance value was equal to that of the focus voxel (i.e., $\mathbb{D}(i)=\mathbb{D}(\mathbb{P}(j)))$. When this condition was true, all voxels which had the ancestor, $\mathbb{P}(j)$, took on the new ancestor, $\mathbb{P}(i)$.
Further measures could be taken to prevent over-segmentation of the void phase, and this logic can be either placed within the primary sweep of ranked voxels or as a secondary sweep of identified pores, including throat radius values in the decision logic. However, no additional measures were performed for this study.

This algorithm summarized above is achieved with the following logical steps beginning at voxel $i$ where $\mathbb{R}(i)=0$ :

For each $\mathrm{j}$ of the 26 neighbors of voxel $\mathrm{i}$

If $\mathbb{B}(\mathrm{i})=\mathbb{B}(\mathrm{j})=0 \|$ If both the focus voxel and the neighbor voxel are void

If $\mathbb{R}(\mathrm{i})<\mathbb{R}(\mathrm{j}) \& \mathbb{P}(\mathrm{j})=\mathbb{I}$ (j) \|If the rank of the focus voxel supersedes the rank of the neighbor, and if the neighbor has not yet been absorbed into another pore

$\mathbb{P}(\mathrm{j})=\mathbb{P}(\mathrm{i}) \|$ Absorb the neighbor into the pore of the focus voxel.

End

If $\mathbb{D}(\mathrm{i})=\mathbb{D}(\mathbb{P}(\mathrm{j})) \|$ If the distance value at the focus equals the distance value of the neighbor's current ancestor

$\mathbb{P}($ find $(\mathbb{P}=\mathbb{P}(\mathrm{j}))=\mathbb{P}(\mathrm{i}) \|$ Reassign the ancestor of the neighbor's pore to that of the focus End End

End

Procedure 1. Algorithm used to reach a segmented state.

This was repeated for $i$, where $\mathbb{R}(i)=1$, then again for $i$, where $\mathbb{R}(i)=2$. This process was repeated while $\mathbb{R}(i)<n$.

Figures 2 contains a 2D demonstration of the matrices involved in the segmentation process, including a finalized $\mathbb{P}$ matrix with the pore voxels outlined. In addition to the $\mathbb{P}$ matrix, the following pore characteristics were then found at the end of the pore segmentation algorithm:

- Pore radius was defined as the maximum value of $\mathbb{D}$ within that pore.

- Pore coordinates were defined as the $x, y, z$ values of the position of the maximal $\mathbb{D}$ value.

- Pore volume was defined as the number of voxels within that pore, multiplied by the voxel volume.

Throat isolation and characterization.-Due to the nature of the pore segmentation sequence, pores interfaced with each other at surfaces containing the three-dimensional saddle points in $\mathbb{D}$. These saddle points are the points between the pores that define the size of the largest sphere that could pass from pore to pore. The voxels at and around the saddle point were analyzed for throat radius calculation as follows.

A list of potential throat voxels was created by finding all voxels, $i$, that have a $\mathbb{D}>1$, that were also neighbors to at least one voxel, $j$, such that $\mathbb{P}(j) \neq \mathbb{P}(i)$ and $\mathbb{P}(j) \neq 0$. For all potential throat voxels, a list of its 26 neighbors in $\mathbb{P}$ was compiled. If the two most frequent non-negative values present constituted at least 12 of the 26 neighbors, and one value did not constitute more than 21 of the 26 neighbors, that voxel was added to a list of throat voxels connecting the two dominant pores. The above criteria were used to minimize the number of unwanted connections made between pores with irregular shapes. This list was then evaluated on the non-filtered distance map, $\mathbb{D}$, to determine the throat diameter. For completeness, the algorithm procedure of this throat extraction is provided below (see Procedure 2). 
For each pore voxel $\mathrm{j}$

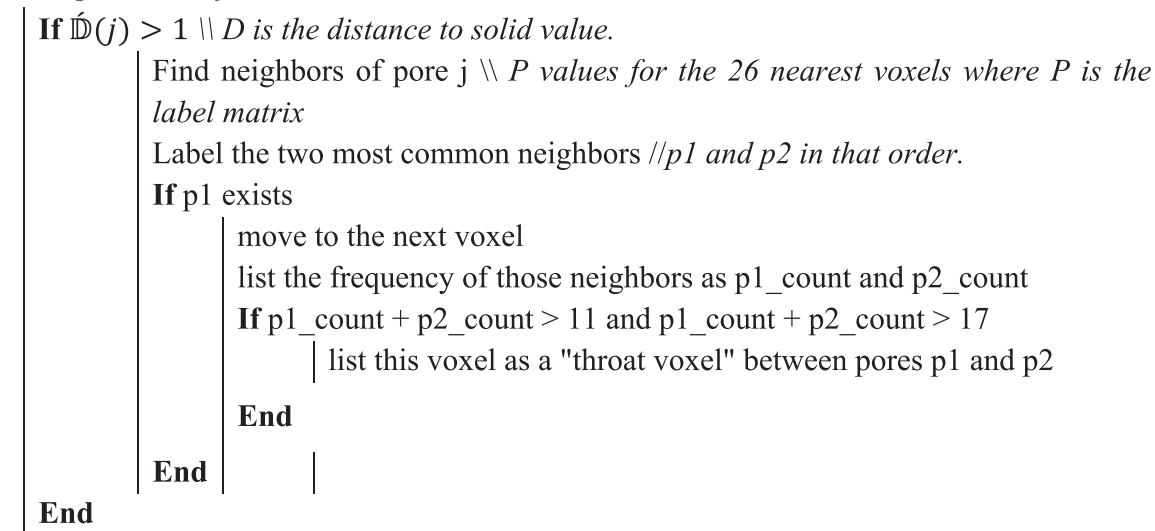

Procedure 2. Algorithm used to segment the throat voxels.

Particular attention was directed to the throat diameter, since the throat diameter plays a significant role in the mass transport calculation (both using invasion percolation or diffusive process). To prevent the discretized values in $\mathbb{D}$ from resulting in only a few, discrete throat diameters, the diameter of each throat $i$, was calculated as follows:

$$
r(i)=\frac{n_{1} r_{1}+n_{2} r_{2}}{n_{1}+n_{2}}+\left(r_{1}-r_{2}\right)
$$

where $r_{1}$ and $r_{2}$ are the two largest unique numbers in the list of voxels evaluated on $\mathbb{D}$ for a given throat, $n_{1}$ is the number of voxels in the list with a $\mathbb{D}$ value of $r_{1}$, and $n_{2}$ is the number of voxels in the list with a $\mathbb{D}$ value of $r_{2}$. This resulted in a radius value slightly larger than $r_{1}$; however, this radius value was ranked in a way that made high aspect ratio throats slightly larger than those with aspect ratios closer to 1 . This throat diameter calculation is fully detailed in Procedure 3.

Several hours on a regular desktop computer are required to extract the pore network (depending on the domain size). Diffusion calculations are completed within seconds.
Diffusion simulation.-Once the pore characteristics (sizes and location) and connectivities were extracted from the CT images of the GDL, the diffusive transport within the GDL pore space was defined. The molar flux of oxygen between two pores connected by a throat (see Figure 3) can be written as,

$$
N_{1,2}=g_{1,2}\left(x_{P_{1}}-x_{P_{2}}\right),
$$

where $N_{1,2}$ is the molar rate between pores 1 and $2, g_{1,2}$ is the diffusive conductance between pores 1 and 2 , and $x_{P_{1}}$ and $x_{P_{2}}$ are the mole fractions of pore 1 and 2, respectively. The diffusive conductance was based on the pore and throat conductances as follows,

$$
g_{1,2}=\left(\frac{1}{g_{t}}+\frac{1}{g_{P_{1}}}+\frac{1}{g_{P_{2}}}\right)^{-1},
$$

where $g_{P_{1}}$ and $g_{P_{2}}$ are the pore conductance of pores 1 and 2, respectively, and $g_{t}$ is the conductance of the connecting throat. The pore

For each throat voxel $\mathrm{j}$

List all $\mathbb{D}(j)$ values for each throat voxel // List the Euclidian distance of each throat

voxel between the connected pores pi and $p j$

$\mathrm{r}_{1}$ and $\mathrm{r}_{2} \leftarrow$ label the two maximum unique $\mathrm{D}$ values // Find the maximum radii

$\mathrm{n}_{1}$ and $\mathrm{n}_{2} \leftarrow$ count the occurrences of $\mathrm{r}_{1}$ and $\mathrm{r}_{2}$ in the list $\mathbb{D}(j) / /$ count the occurrence of

the maximum radii in the list of Euclidian distance for each throat voxels

Throat diameter $\leftarrow$ Apply Equation 3 // compute the throat diameter

End

Procedure 3. Algorithm used to compute the throat diameter.

Pore network analysis.-Once the pore and throat networks were extracted from the segmented images, the networks were analyzed with respect to the pore and throat size distributions. This analysis was performed by fitting the pore and throat size distributions to the log-normal distribution, similar to Zenyuk et al. ${ }^{28}$ Unimodal distributions were chosen as suggested by Zenyuk et al. ${ }^{28}$ for GDL samples compressed using large stains (higher than 0.20).

$$
y=\frac{A}{x \sigma \sqrt{2 \pi}} e^{-\frac{(\ln x-\mu)^{2}}{2 \sigma^{2}}},
$$

where the histogram area, $A$, the mean, $\mu$, and the standard deviation, $\sigma$, were numerically fitted onto the extracted pore and throat size distribution. These parameters were then used to compute the average pore and throat diameters, $\bar{d}$, as well as their standard deviation, $d_{s t d}$, as follows:

$$
\begin{gathered}
\bar{d}=e^{\left(\mu+\sigma^{2}\right) / 2}, \\
d_{s t d}=e^{\left(2 \mu+\sigma^{2}\right)}\left(e^{\sigma^{2}}-1\right) .
\end{gathered}
$$

conductances were defined based on their structural properties, which were calculated during the pore network extraction as,

$$
g_{P_{1,2}}=c_{t o t} D_{b} \frac{A_{p_{1,2}}}{R_{p_{1,2}}},
$$

where $c_{\text {tot }}$ is the total air molar concentration, $D_{b}$ is the bulk diffusivity, $A_{p_{i}}$ is the cross sectional area of the pore $i$, and $R_{p_{i}}$ is the radius of

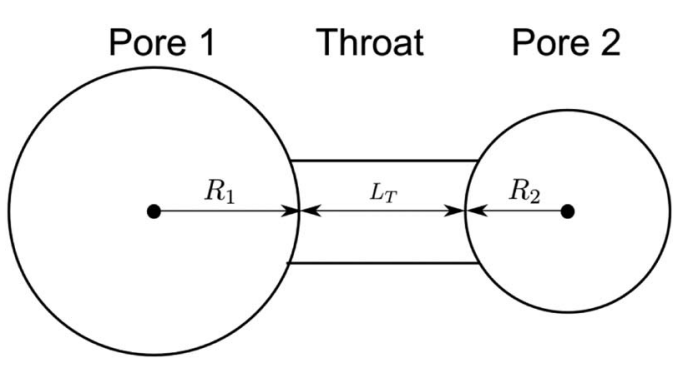

Figure 3. Schematic of two pores connected by a throat 


\begin{tabular}{|c|c|c|}
\hline Name & Unit & Value \\
\hline & $\mathrm{mol} / \mathrm{m}^{3}$ & 40.89 \\
\hline$D_{\text {bulk }}$ & $\mathrm{mm}^{2} / \mathrm{s}$ & $20.68^{48}$ \\
\hline$x_{i n}$ & - & 1 \\
\hline$x_{\text {out }}$ & - & 0 \\
\hline
\end{tabular}

the pore $i$. In Equation 9, a small pore has a small cross sectional area which leads to a small conductance. The throat conductance, $g_{t}$, was defined in a similar way as,

$$
g_{t}=c_{t o t} D_{b} \frac{A_{t}}{L_{t}},
$$

where $A_{t}$ is the throat cross sectional area, and $L_{t}$ is the throat length between the two considered pores. The throat length is defined as the length between two neighboring pores (see Figure 3). The routine, OpenPNM. Geometry.models.throat_length.straight(network = pn,geometry $=$ geom), was used to compute the length between two pore centers less their respective diameters (as illustrated in Figure 3). It is worth noting that in Equation 10 for the case of narrow and long throats, i.e. small $A_{t}$ and/or large $L_{t}$, the throat conductance, $g_{t}$, may be small compared to the pore conductance. This relative difference could lead to the dominance of the throat size on the total conductance. Therefore characterizing the throat size becomes critical for accurately modelling the GDL effective diffusivity.

Equations 7 to 10 were applied to all the pores in the extracted network using OpenPNM, ${ }^{16}$ which led to a linear system of equations where the mole fraction of oxygen within the pore space are the unknowns. Once the linear system was solved, the effective diffusivity of the GDL, $D^{e f f}$, was computed as follows:

$$
D^{e f f}=-\frac{N_{\text {tot }} L}{\left(x_{\text {in }}-x_{\text {out }}\right) c_{\text {tot }} A}
$$

where $N_{\text {tot }}$ is the total molar flow rate entering in the GDL calculated by OpenPNM (in mol/s), $L$ is the thickness of the GDL, $A$ is the crosssectional area of the GDL, and $x_{i n}$ and $x_{\text {out }}$ are the inlet and outlet molar fractions, respectively, defined as the boundary conditions at the extremities of the GDL. The parameters used in our simulation are given in Table II.

\section{Results and Discussion}

GDL structural analysis. - The through-plane porosity distribution of the segmented images was first analyzed for each GDL sample. The reduction in thickness resulted in decreases in porosity for both the SGL 25AA and SGL 34AA GDLs (Figures $4 \mathrm{a}$ and 4b). The uncompressed thicknesses reported in Table I correspond to the porosities where $\varepsilon<0.95$. Each of the porosity profiles presented a wavy-like pattern with a minimum of porosity close to the extremities (e.g., at $-100 \mu \mathrm{m}$ and $75 \mu \mathrm{m}$ for the uncompressed 34AA GDL). Such wavy-like patterns were also observed by Fishman et al. ${ }^{49}$ for the SGL series GDLs. By increasing the compression of the GDL, the porosity distributions became increasingly flat in the GDL core. This was particularly apparent in the 34AA sample at compressive strains of 0.40 , 0.50 , and 0.60 (Figure $4 b$ ).

For a given compressive strain, the measured porosity of SGL 25AA was lower than SGL 34AA. This trend is in agreement with the specifications given by the manufacturer for SGL 25 and 34 series GDLs, as well as with the literature. ${ }^{36}$ In Figure 4c, the mean throughplane porosity distributions are presented versus the compressive strain of the sample. As previously mentioned, the SGL 25AA GDL samples were less porous than the SGL 34AA GDL samples. This justified the need to go to higher strains for the SGL 25AA GDL in order to significantly decrease the GDL porosity.

In Rashapov et al., ${ }^{36}$ it was reported that the porosity in a compressed sample can be obtained with $\varepsilon=1-l_{0}\left(1-\varepsilon_{0}\right) / l$, where $\varepsilon_{0}$
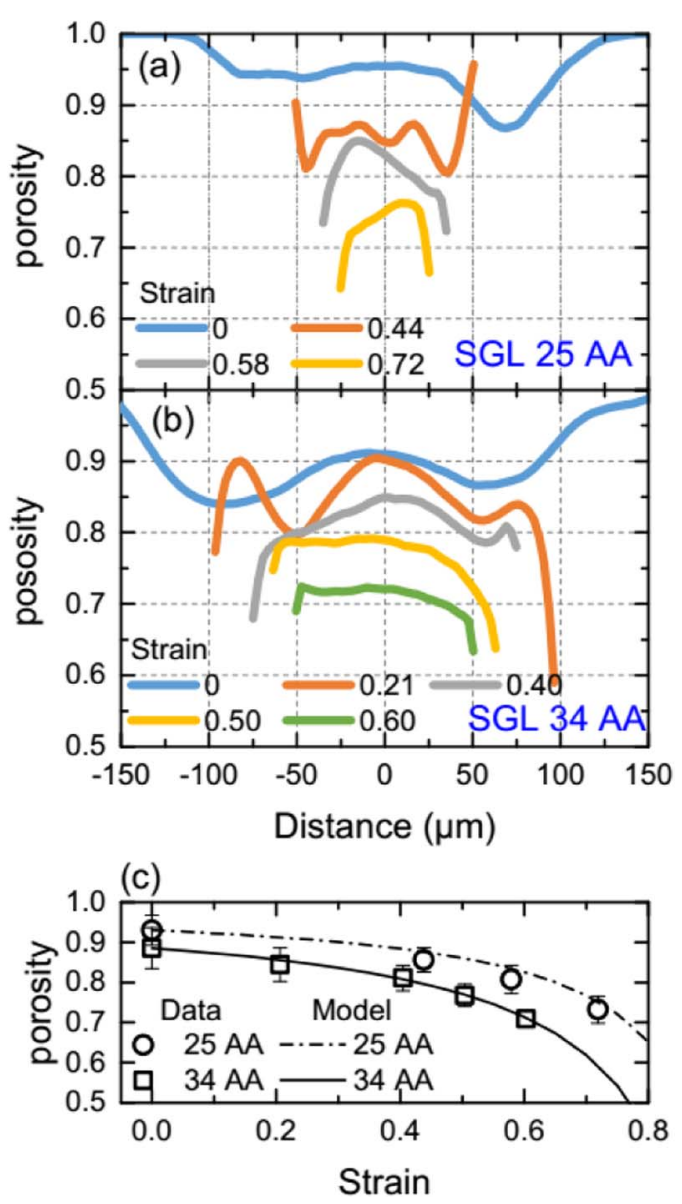

Figure 4. Through-plane porosity distributions for the SGL 25AA GDL (a) and SGL 34AA GDL (b). (c), Mean porosity versus the sample compressive strain. The model is computed using Equation 12.

is the initial thickness. Using the definition of the strain given in CT and segmentation section, this expression leads to the following:

$$
\varepsilon(s)=1-\frac{1}{1-s}\left(1-\varepsilon_{0}\right) .
$$

The comparison of Equation 12 to the porosity of the compressed sample versus strain is presented in Figure $4 \mathrm{c}$, and excellent agreement between the model and the experimental data was observed for both GDL samples, thereby validating our methodology used to segment the CT images.

Pore network analysis.-The equivalent pore network was extracted using the algorithm described in Section 2.2. The void space of each GDL binary tomogram (Figure 5a) was divided into pores. A resulting image of the pore network of the uncompressed SGL 34AA GDL is presented in Figure 5b, where each color represents a distinct pore, and the fibers were colored in white. The void space between two fibers in Figure 5a was divided into multiple pores due to the presence of other fibers in the neighboring through-plane positions. In addition to the pore location, the extraction algorithm was also used to compute the pore diameter, volume, and connectivities of the neighboring pores. The throats (not depicted in Figure 5b) were extracted from the binary images, and their diameter and connectivity were also computed, as discussed in Section 2.2. This data was used to define the conductivity between each pore in the network, according to Equations 6 to 9 .

The pore network was then analyzed with respect to the pore and throat diameter distribution produced via the pore network segmentation. The frequency of pore and throat diameters were computed over a range spanning from 1 to $150 \mu \mathrm{m}$ every $5 \mu \mathrm{m}$ for all GDL 
(a)

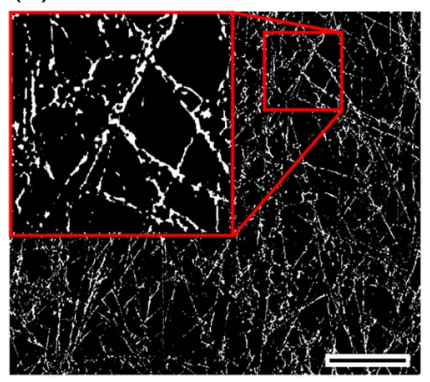

(b)

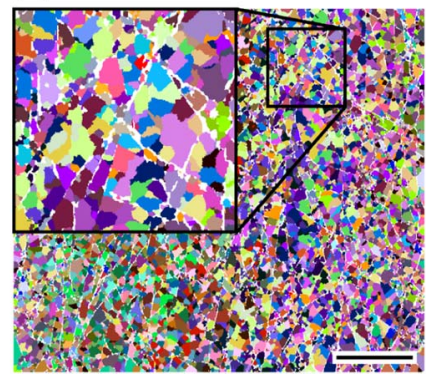

Figure 5. (a), Segmented image of the uncompressed SGL 34AA GDL. (b), Equivalent pore network. The length of the scale bar is $1 \mathrm{~mm}$.

samples. The resulting distributions are presented in Figures $6 \mathrm{a}$ to $6 \mathrm{~d}$ and fitted to the log-normal law presented in Section 2.2. All parameters obtained from the curve fitting are presented in Table I in the supplementary information section. As the compressive strain increased, the mean and standard deviations of the pores and throats decreased for both GDL series. This effect was expected due to the compression of the GDL samples which reduced the void spaces between fibers, resulting in smaller pore diameters.

To characterize pore diameter distributions, a pore network extraction is not necessarily required. Mercury porosimetry invasion (MIP) techniques can also be used to characterize the internal structure of a porous material. However, throat size distributions are not easily measurable, even though throat sizes provide critical data necessary to study fluid transport within the porous media. As such, there is a great advantage to using a pore network extraction algorithm based on CT images to compute the throat size distribution of the porous domain. Using the parameters reported in Table I from the supplementary material section, and the log-normal distribution (Equation 3), the pore and throat diameter distributions can be reconstructed for the SGL 25AA and SGL 34AA GDLs for a range of compressive strains.

The effect of the compressive strain on the mean GDL pore and throat diameters was analyzed in Figures 6e and $6 f$ for both GDLs. The mean pore diameter was observed to linearly decrease with higher compressive strain, an effect already described by Zenyuk et al. ${ }^{28}$ In the SGL 25AA GDL sample, the mean pore diameter decreased from $53 \mu \mathrm{m}$ to $28 \mu \mathrm{m}$. In the SGL 25AA GDL sample, the mean pore diameter decreased from $53 \mu \mathrm{m}$ to $28 \mu \mathrm{m}$. In comparison with increasing compressive strain, the SGL 34AA GDL mean pore diameters were smaller than the SGL 25AA GDL pores, ranging from $38 \mu \mathrm{m}$ to $29 \mu \mathrm{m}$. In Figure $6 \mathrm{c}$, the slopes of the linear curves of the mean pore diameter were observed to be $-22 \mu \mathrm{m}^{-1}$ and $-17 \mu \mathrm{m}^{-1}$ for SGL 34AA and SGL 25AA, respectively, indicating that the SGL 25AA GDL was more sensitive to compression than SGL 34AA. In contrast, the mean throat diameters were found to be relatively constant regardless of the applied compressive strain on both GDL samples. These results may indicate that the compression of the GDL samples had a significant impact on the average pore diameters (but not on the throat diameters).

Effective diffusivity calculation.-The inlet (left side) and outlet (right side) oxygen mole fractions were arbitrarily defined as 1 and 0 , respectively. Note that the value of these boundary conditions did not change the results of the effective diffusivity calculations. A linear system of $\mathrm{N}$-equations (corresponding to the $\mathrm{N}$-connectivities that
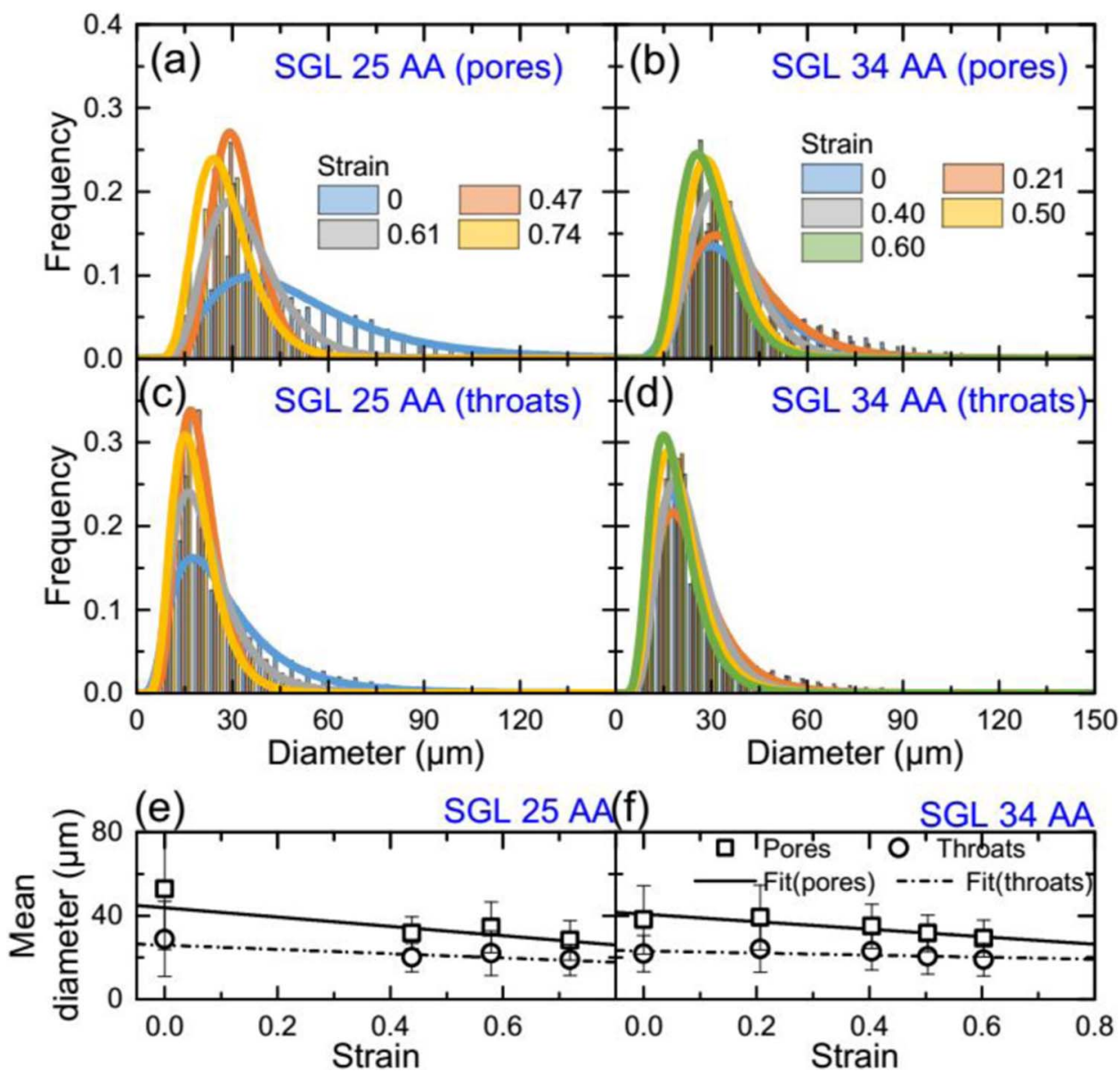

Figure 6. Pore and throat diameter distributions for the SGL 25AA GDL samples (a) and (b), and the SGL 34AA GDL samples (c) and (d). Mean pore and throat diameters versus compressive strain for the SGL 25AA GDL samples (e) and SGL 34AA GDL sample (f). 
(a)

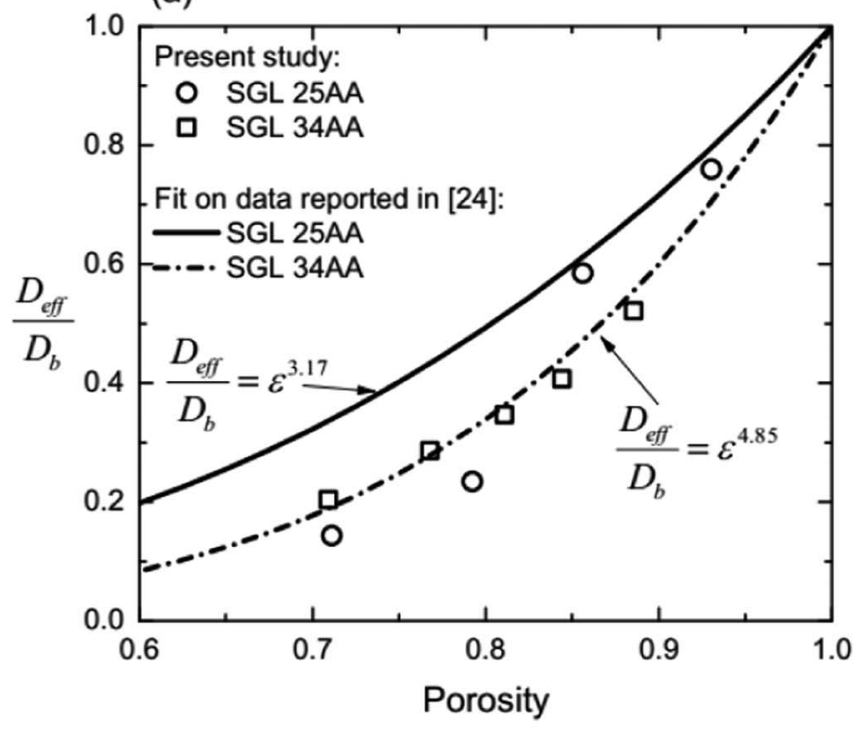

(b)

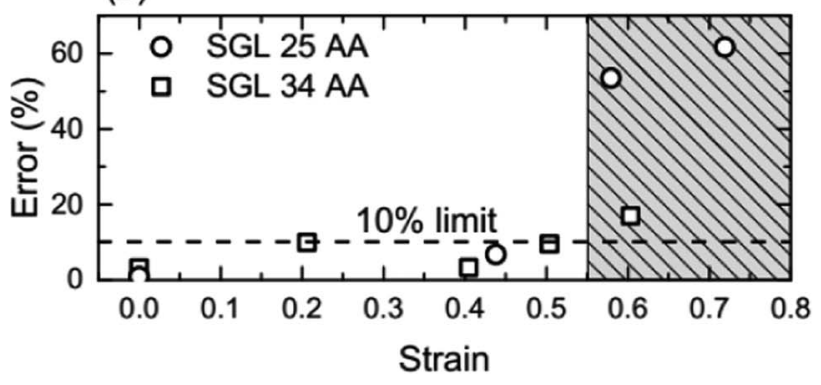

Figure 7. (a), Dry dimensionless diffusivity as a function of material porosity for the SGL 25 AA and SGL 34 AA GDLs. (b), Relative error between the experimental data measured by Rashapov et al. ${ }^{36}$ and the numerical results obtained from OpenPNM.

exist within the pore network) was solved using the pore network modeling package OpenPNM. ${ }^{16}$ The mole fraction in each of the pores of the network was similarly computed. A result of these calculations is given in the supplementary materials, where the map of concentration is presented. This shows that the local information (such as pore molar concentration) can also be obtained using our PNM extraction.

The molar flow rates in all of the throats of the network, which depended on the local pore and throat structure (see Equation 6), were also computed. The total molar flux flowing through the network was then computed at the inlet and used to calculate $D^{e f f}$ using Equation 11. All of these calculations were applied to the nine GDL samples considered in this study.

The normalized diffusivities (effective diffusivity divided by the bulk diffusivity) are presented in Figure 7a for the nine GDL samples considered in this study. These results are compared with the data presented by Rashapov et al. ${ }^{36}$ For the sake of clarity, the IP effective diffusivity measurements as a function of porosity reported by Rashapov et al. for SGL 25AA and SGL 34AA GDLs were used and fitted to a power law. The effective diffusivities computed from the pore network extractions are in good agreement for both GDLs, with the exception of SGL 25AA GDL samples that underwent a compressive strain of 0.58 and 0.72 . These discrepancies are discussed in the next paragraph. The computed diffusivity for the SGL 25AA GDL was higher than that for the SGL 34AA GDL. We attribute this difference to the related structural differences. For example, the SGL 25AA GDL is thinner and more porous (see GDL structural analysis and Pore network analysis sections) than the SGL 34AA GDL, allowing for an increased rate of gas transport through the SGL 25AA GDL. In a similar way, decreasing the GDL porosity resulted in a decreased mean pore diameter, which also led to a significant decrease in the effective diffusivity. Therefore, the computed effective diffusivity presented in this study was in good agreement with the literature and experimental measurements, thereby validating our combined approach of watershed-derived pore networks and computed tomography for characterizing fuel cell GDL effective diffusivity.

In Figure $7 b$, the relative error between the computed effective diffusivity presented in this study, and the fit obtained from the experimental measurements reported in Ref. 36 are compared with respect to the compressive strain of each sample. A relatively low error (below $10 \%$ ) was found for all the samples compressed with a compressive stain less than 0.55 . Above this value, the relative error significantly increased to $60 \%$. Therefore, our results indicate that a limit exists to the applicability of our method. By highly compressing our GDLs (compressive strains greater than 0.55), the sizes of the pore diameters approached the resolution of the CT scanner used in this study (a few microns). At these extremes, the structure of these small pores cannot be adequately captured from microscale CT images. ${ }^{42}$ The structural characteristics of the highly compressed GDLs may not have been fully captured during network extraction process, leading to significant errors in the predicted effective diffusivity of oxygen. Therefore, a sub-micron resolution CT scanner is recommended for studying the transport properties of GDLs that have undergone high compressive strains. Finally, it can be noted that for a given strain of 0.6 , for example, this effect was more pronounced for the SGL 25AA GDL (71 $\mu \mathrm{m}$ thick) compared to SGL 34AA GDL (100 $\mu \mathrm{m}$-thick). At this high strain, SGL 34AA retained larger pore sizes compared to SGL 25AA.

\section{Conclusions}

In this study, a pore network extraction methodology to predict the transport properties of fuel cell GDLs was used. This methodology was based on the pore network modelling of oxygen diffusion, where the pore network was extracted from CT images of commercial GDLs. Two sets of GDLs, namely SGL 25AA and SGL 34AA, were examined over a range of compression ratios (or strain). The structural properties, porosity distributions, pore and throat diameter distributions of the GDL, were extracted and analyzed. These results showed that the significant compression of a GDL led to a decrease in pore diameters. However, this pore size decrease did not have a significant impact on the throat diameters of the GDL.

The pore networks of compressed and uncompressed GDLs were reconstructed, and the effective diffusivities were computed using the open source package, OpenPNM. ${ }^{16}$ Our computations were compared with experimental data from the literature for the same set of GDLs, and good agreement with the experimental data was found, thereby validating our approach for IP effective diffusivity measurements. We also observed that the accuracy of our effective diffusivity calculations was linked to the resolution of the CT images, where the analysis of highly compressed GDLs with relatively small pores ( $\sim 5$ to $10 \mu \mathrm{m}$ in diameter) would benefit from a CT resolution of at least $3 \mu \mathrm{m}$.

To conclude, this study reports an alternative pore network extraction methodology which is versatile and customizable. This tool can be used to characterize easily IP effective diffusivity from CT of thin porous layer such as GDL. Although this GDL properties is critical for designing GDL thickness and channel to rib ratio, it was rarely studied in the past literature. Our work fills this gap, and brings a methodology to build a watershed-derived pore network extraction algorithm. Very importantly, we validated the accuracy of watershed-derived pore networks for predicting in-plane effective diffusivities, further establishing the reliability of watershed-derived pore network modeling for fuel cell applications.

\section{Acknowledgments}

Financial support from the Natural Sciences and Engineering Research Council of Canada (NSERC) Discovery Grants Program 
and the NSERC Canada Research Chairs Program are gratefully acknowledged.

\section{ORCID}

\section{A. Bazylak (D) https://orcid.org/0000-0002-9594-4930}

\section{References}

1. M. M. Mench, ed., Fuel Cell Engines, John Wiley \& Sons, Inc., Hoboken, NJ, USA, 2008.

2. J. P. Owejan, J. J. Gagliardo, J. M. Sergi, S. G. Kandlikar, and T. A. Trabold, "Water management studies in PEM fuel cells, Part I: Fuel cell design and in situ water distributions," Int. J. Hydrogen Energy., 34, 3436 (2009).

3. C. Ziogou, S. Voutetakis, M. C. Georgiadis, and S. Papadopoulou, "Model predictive control (MPC) strategies for PEM fuel cell systems - A comparative experimental demonstration," Chem. Eng. Res. Des., 131, 656 (2018).

4. T. Yoshida and K. Kojima, "Toyota MIRAI Fuel Cell Vehicle and Progress Toward a Future Hydrogen Society," Interface Mag. 24, 45 (2015).

5. D. Hayashi, A. Ida, S. Magome, T. Suzuki, S. Yamaguchi, and R. Hori, Synchrotron $X-R a y$ Visualization and Simulation for Operating Fuel Cell Diffusion Layers, in: 2017.

6. M. Srinivasarao, D. Bhattacharyya, R. Rengaswamy, and S. Narasimhan, "Multivariable optimization studies of cathode catalyst layer of a polymer electrolyte membrane fuel cell," Chem. Eng. Res. Des., 89, 10 (2011).

7. M. F. Mathias, J. Roth, J. Fleming, and W. Lehnert, "Diffusion media materials and characterisation," Handb. Fuel Cells., 3, 517 (2010).

8. Y. Wang, K. S. Chen, J. Mishler, S. C. Cho, and X. C. Adroher, "A review of polymer electrolyte membrane fuel cells: Technology, applications, and needs on fundamental research," Appl. Energy., 88, 981 (2011).

9. J. M. Morgan and R. Datta, "Understanding the gas diffusion layer in proton exchange membrane fuel cells. I. How its structural characteristics affect diffusion and performance," J. Power Sources., 251, 269 (2014).

10. S. Chevalier, J. Lee, N. Ge, R. Yip, P. Antonacci, Y. Tabuchi, T. Kotaka, and A. Bazylak, "In operando measurements of liquid water saturation distributions and effective diffusivities of polymer electrolyte membrane fuel cell gas diffusion layers," Electrochim. Acta., 210, 792 (2016).

11. M. Fazeli, J. Hinebaugh, and A. Bazylak, "Incorporating Embedded Microporous Layers into Topologically Equivalent Pore Network Models for Oxygen Diffusivity Calculations in Polymer Electrolyte Membrane Fuel Cell Gas Diffusion Layers," Electrochim. Acta., 216, 364 (2016).

12. M. Fazeli, J. Hinebaugh, Z. Fishman, C. Tötzke, W. Lehnert, I. Manke, and A. Bazylak, "Pore network modeling to explore the effects of compression on multiphase transport in polymer electrolyte membrane fuel cell gas diffusion layers," J. Power Sources. 335, 162 (2016).

13. T. G. Tranter, P. Stogornyuk, J. T. Gostick, A. D. Burns, and W. F. Gale, "A method for measuring relative in-plane diffusivity of thin and partially saturated porous media: An application to fuel cell gas diffusion layers," Int. J. Heat Mass Transf., 110, 132 (2017).

14. A. Bazylak, "Liquid water visualization in PEM fuel cells: A review," Int. J. Hydrogen Energy., 34, 3845 (2009).

15. S. Chevalier, C. Josset, and B. Auvity, "Fluid dynamic breakthrough in two connected capillaries: From stationary to oscillating state," Phys. Fluids., 29, 102102 (2017).

16. J. Gostick, M. Aghighi, J. Hinebaugh, T. Tranter, M. A. Hoeh, H. Day, B. Spellacy, M. H. Sharqawy, A. Bazylak, A. Burns, W. Lehnert, and A. Putz, "OpenPNM: A Pore Network Modeling Package," Comput. Sci. Eng., 18, 60 (2016).

17. T. G. Tranter, J. T. Gostick, A. D. Burns, and W. F. Gale, "Pore Network Modeling of Compressed Fuel Cell Components with OpenPNM," Fuel Cells. 16, 504 (2016)

18. B. Markicevic, A. Bazylak, and N. Djilali, "Determination of transport parameters for multiphase flow in porous gas diffusion electrodes using a capillary network model," J. Power Sources., 171, 706 (2007).

19. J. Hinebaugh, A. Bazylak, and P. P. Mukherjee, Multi-scale modeling of two-phase transport in polymer electrolyte membrane fuel cells, in: Polym. Electrolyte Membr. Direct Methanol Fuel Cell Technol., Elsevier, 2012: p. 254.

20. M. Aghighi, M. A. Hoeh, W. Lehnert, G. Merle, and J. Gostick, "Simulation of a Full Fuel Cell Membrane Electrode Assembly Using Pore Network Modeling," J. Electrochem. Soc., 163, F384 (2016).

21. J. T. Gostick, "Random Pore Network Modeling of Fibrous PEMFC Gas Diffusion Media Using Voronoi and Delaunay Tessellations," J. Electrochem. Soc., 160, F731 (2013).

22. R. Wu, X. Zhu, Q. Liao, R. Chen, and G.-M. Cui, "Liquid and oxygen transport in defective bilayer gas diffusion material of proton exchange membrane fuel cell," Int. J. Hydrogen Energy., 38, 4067 (2013).

23. R. Wu, X. Zhu, Q. Liao, H. Wang, Y. Ding, J. Li, and D. Ye, "Determination of oxygen effective diffusivity in porous gas diffusion layer using a three-dimensional pore network model," Electrochim. Acta., 55, 7394 (2010).
24. P. Čapek, V. Hejtmánek, L. Brabec, A. Zikánová, and M. Kočiřík, "Effective diffusivities of gases in a reconstructed porous body," Chem. Eng. Res. Des., 86, 713 (2008).

25. J. T. Gostick, M. A. Ioannidis, M. W. Fowler, and M. D. Pritzker, "Pore network modeling of fibrous gas diffusion layers for polymer electrolyte membrane fuel cells," J. Power Sources., 173, 277 (2007).

26. G. Luo, Y. Ji, C.-Y. Wang, and P. K. Sinha, "Modeling liquid water transport in gas diffusion layers by topologically equivalent pore network," Electrochim. Acta., 55, $5332(2010)$.

27. T. Agaesse, A. Lamibrac, F. N. Büchi, J. Pauchet, and M. Prat, "Validation of pore network simulations of ex-situ water distributions in a gas diffusion layer of proton exchange membrane fuel cells with X-ray tomographic images," J. Power Sources., 331, 462 (2016)

28. I. V. Zenyuk, D. Y. Parkinson, L. G. Connolly, and A. Z. Weber, "Gas-diffusion-layer structural properties under compression via X-ray tomography," J. Power Sources., 328, 364 (2016).

29. J. T. Gostick, "Versatile and efficient pore network extraction method using markerbased watershed segmentation," Phys. Rev. E., 96, 023307 (2017).

30. N. Zamel and X. Li, "Effective transport properties for polymer electrolyte membrane fuel cells - With a focus on the gas diffusion layer," Prog. Energy Combust. Sci., 39, 111 (2013).

31. S. Chevalier, J.-C. Olivier, C. Josset, and B. Auvity, "Polymer Electrolyte Membrane Fuel Cell Characterisation Based on Current Distribution Measurements," ECS Trans. 86, 211 (2018).

32. D. R. Baker, D. A. Caulk, K. C. Neyerlin, and M. W. Murphy, "Measurement of Oxygen Transport Resistance in PEM Fuel Cells by Limiting Current Methods," $J$. Electrochem. Soc., 156, B991 (2009).

33. P. Antonacci, S. Chevalier, J. Lee, R. Yip, N. Ge, and A. Bazylak, "Feasibility of combining electrochemical impedance spectroscopy and synchrotron X-ray radiography for determining the influence of liquid water on polymer electrolyte membrane fuel cell performance," Int. J. Hydrogen Energy., 40, 16494 (2015).

34. R. Flückiger, S. A. Freunberger, D. Kramer, A. Wokaun, G. G. Scherer, and F. N. Büchi, "Anisotropic, effective diffusivity of porous gas diffusion layer materials for PEFC," Electrochim. Acta., 54, 551 (2008).

35. P. Mangal, L. M. Pant, N. Carrigy, M. Dumontier, V. Zingan, S. Mitra, and M. Secanell, "Experimental study of mass transport in PEMFCs: Through plane permeability and molecular diffusivity in GDLs," Electrochim. Acta., 167, 160 (2015).

36. R. Rashapov, F. Imami, and J. T. Gostick, "A method for measuring in-plane effective diffusivity in thin porous media," Int. J. Heat Mass Transf., 85, 367 (2015).

37. N. Zamel, N. G. C. Astrath, X. Li, J. Shen, J. Zhou, F. B. G. Astrath, H. Wang, and Z.-S. Liu, "Experimental measurements of effective diffusion coefficient of oxygen-nitrogen mixture in PEM fuel cell diffusion media," Chem. Eng. Sci., 65, 931 (2010).

38. P. A. García-Salaberri, J. T. Gostick, G. Hwang, A. Z. Weber, and M. Vera, "Effective diffusivity in partially-saturated carbon-fiber gas diffusion layers: Effect of local saturation and application to macroscopic continuum models," J. Power Sources., 296, 440 (2015).

39. S. Chevalier, N. Lavielle, B. D. Hatton, and A. Bazylak, "Novel electrospun gas diffusion layers for polymer electrolyte membrane fuel cells: Part I. Fabrication, morphological characterization, and in situ performance," J. Power Sources., 352, 272 (2017).

40. R. R. Rashapov and J. T. Gostick, In-Plane Effective Diffusivity in PEMFC Gas Diffusion Layers, Transp. Porous Media. 1 (2016).

41. D. Kramer, S. A. Freunberger, R. Flückiger, I. A. Schneider, A. Wokaun, F. N. Büchi, and G. G. Scherer, "Electrochemical diffusimetry of fuel cell gas diffusion layers," J. Electroanal. Chem., 612, 63 (2008).

42. R. Banerjee, J. Hinebaugh, H. Liu, R. Yip, N. Ge, and A. Bazylak, "Heterogeneous porosity distributions of polymer electrolyte membrane fuel cell gas diffusion layer materials with rib-channel compression," Int. J. Hydrogen Energy., (2016).

43. A. El-kharouf, T. J. Mason, D. J. L. Brett, and B. G. Pollet, "Ex-situ characterisation of gas diffusion layers for proton exchange membrane fuel cells," J. Power Sources., 218, 393 (2012).

44. R. R. Rashapov, J. Unno, and J. T. Gostick, "Characterization of PEMFC Gas Diffusion Layer Porosity," J. Electrochem. Soc., 162, F603 (2015).

45. H. Dong and M. J. Blunt, "Pore-network extraction from micro-computerizedtomography images," Phys. Rev. E., 80, 036307 (2009).

46. S. Beucher, Watersheds of functions and picture segmentation, in: ICASSP '82. IEEE Int. Conf. Acoust. Speech, Signal Process., Institute of Electrical and Electronics Engineers, n.d.: pp. 1928.

47. C. R. Maurer, Rensheng Qi, and V. Raghavan, "A linear time algorithm for computing exact Euclidean distance transforms of binary images in arbitrary dimensions," IEEE Trans. Pattern Anal. Mach. Intell., 25, 265 (2003).

48. E. N. Fuller, P. D. Schettler, and J. C. Giddings, "A new method for prediction of binary gas-phase diffusion coefficients.," Ind. Eng. Chem., 16, 551 (1966).

49. Z. Fishman, J. Hinebaugh, and A. Bazylak, "Microscale Tomography Investigations of Heterogeneous Porosity Distributions of PEMFC GDLs," J. Electrochem. Soc., 157, B1643 (2010). 